\title{
Non-linear behavior of ring-down time in cavity ring-down spectroscopy with tapered fibers
}

\author{
Kavita Sharma ${ }^{1,2^{*}}$, Deepa Venkitesh ${ }^{1}$, Shanti Bhattacharya ${ }^{1}$, Balaji Srinivasan ${ }^{1}$, Gilberto Brambilla ${ }^{2}$ \\ ${ }^{I}$ Department of Electrical Engineering, Indian Institute of Technology Madras, Chennai - 600036, India, \\ ${ }^{2}$ Optoelectronics Research Centre, University of Southampton, Southampton SO17 1BJ, U.K. \\ *ee11d038@ee.iitm.ac.in
}

\begin{abstract}
The effect of surrounding refractive index $\left(n_{2}\right)$ on ring-down time $(\tau)$ is studied in conventional and amplified cavity ring-down spectroscopy with tapered fibers. Simulation and experimental results indicate that $\tau$ varies as rational function of $n_{2}$.

OCIS codes: (280.4788) Optical sensing and sensors; (300.6340) Spectroscopy, infrared.
\end{abstract}

\section{Introduction}

Single mode and multimode tapers have been used widely in different configurations for achieving better sensitivity for refractive index measurements [1,2]. All these techniques use a spectral measurement to quantify the measured parameter. Time domain techniques such as cavity ring-down spectroscopy (CRDS) has been recently proposed to be used in conjunction with tapered fibers because of the advantages that include faster response, relatively low cost, insensitivity to source fluctuations and good sensitivity $[3,4]$.

A theoretical study of the effect of a change in surrounding refractive index on the evanescent field in tapered fibers is crucial to quantify the absorption loss induced by the sample and hence for optimizing the experimental parameters. This estimation was done for a taper immersed in water in the case of a single pass configuration [5]. Here, we numerically evaluate the loss induced by an aqueous solution on a fiber taper and the corresponding ring down times in CRDS configuration. We compare these results with those obtained from experiments for conventional and amplified CRDS.

\section{Theory}

A fiber taper consists of a uniform waist region, connected to un-tapered fiber with two transition regions, as shown in the inset of Fig. 1. The transition region converts the core mode in the un-tapered section into a cladding mode in the waist region. The propagation properties and absorption of the evanescent field of this mode are decided by the refractive index of the surrounding region $\left(n_{2}\right)$. Since the refractive index difference between the taper and the surrounding is large, the eigen value equation governing the propagation of modes in a fiber is solved numerically in its rigorous form (without using the weakly guiding approximation), in order to find the effective refractive index $\left(n_{\text {eff }}\right)$ and propagation constant and hence the exact mode distribution of the supporting mode [6]. The power in evanescent-field $\left(\eta_{c l a d}\right)$ is then calculated as

$$
\eta_{\text {clad }}=\frac{P_{\text {clad }}}{P_{0}}=\frac{\iint_{\text {clad }} S_{z} d A}{\int_{-\infty}^{\infty} S_{z} d A},
$$

where $P_{\text {clad }}$ is the power propagating outside the fiber, $P_{0}$ is the total power and $S_{z}$ is the time-averaged Poynting vector, which is calculated using the exact modal distribution of the electric field [6]. The loss introduced by the sample is calculated independently for the waist and transition regions [5]. The transition region is modeled as having an exponential profile [7] and is discretized with sufficient precision. The total loss $(\alpha)$ is used to calculate the ring-down time ( $\tau$ ) of a CRDS cavity given as $\tau=t_{r} / \alpha$, where $t_{r}$ is the round-trip time of the cavity. The slope of the $\tau$ - $\alpha$ calibration curve decides the sensitivity of a refractometric sensor, where $\alpha$ is decided by $n_{2}$.

\section{Results and discussion}

With water as the surrounding medium, $\left(n_{2}=1.316\right.$ at $\left.1550 \mathrm{~nm}\right)$, the $n_{\text {eff }}$ and $\eta_{\text {clad }}$ are calculated for different taper waist diameters and are shown in Fig 1. Increase in taper diameter leads to a larger $n_{\text {eff; }}$ better confinement of the mode, resulting in a decrease in $\eta_{\text {clad }}$. Thus, smaller taper diameters are preferred for improved sensitivity. The loss introduced by the taper is also dependent on the length of the waist. This dependence is found to be linear, as confirmed through independent simulations. Smaller diameter and longer lengths provides larger loss values, giving rise to better sensitivity.

Aqueous solutions of refractive indices 1.316-1.344 are used to study the effect of surrounding refractive index change. The loss values evaluated numerically are shown as green triangles in Fig. 2, which follows a quadratic 
function as indicated by the fit shown. The change in the corresponding ring-down times $\left(\tau-n_{2}\right.$ curve $)$, calculated for a typical cavity length of $7 \mathrm{~m}$ for conventional CRDS and $16 \mathrm{~m}$ for amplified CRDS are shown in Fig. 3 and 4 as green triangles. The ring-down time varies as a rational function as it is inversely proportional to the loss, unlike the result shown in [3] where a linear dependence is assumed. This would imply that the measurement sensitivity is different at different surrounding refractive indices.

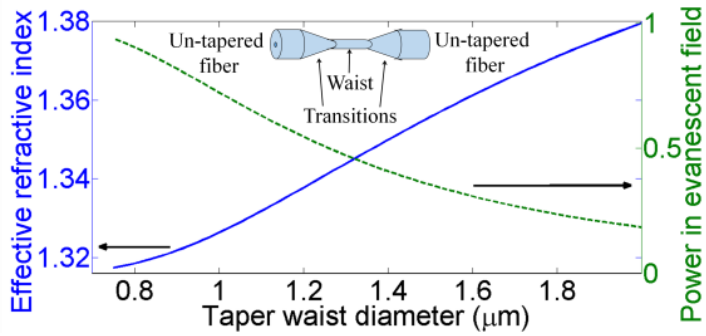

Fig. 1. Effective refractive index and power in evanescent field for different waist diameters. Inset shows the schematic of a taper.

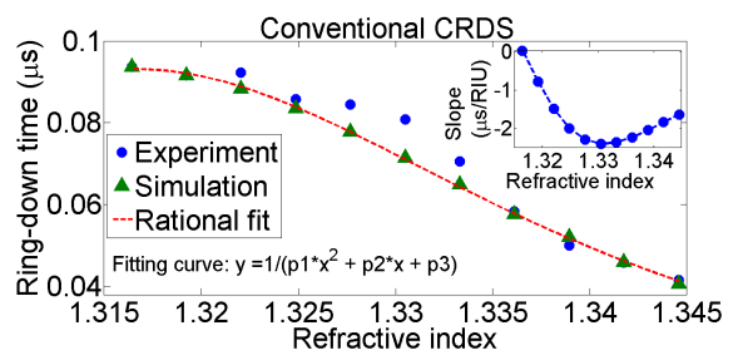

Fig. 3. Change in ring-down time for conventional CRDS experiments along with the simulation results. The inset shows the change in slope with the refractive index.

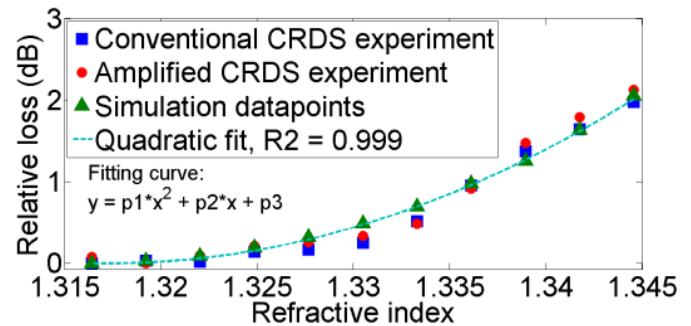

Fig. 2. Relative loss values for conventional and amplified CRDS experiments with simulation results.

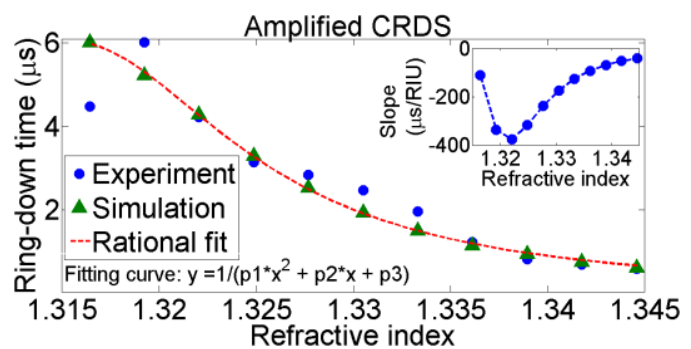

Fig. 4. Change in the ring-down time for amplified CRDS experiments along with the simulation results. The inset shows the change in the slope with refractive index.

Experimental results of conventional and amplified CRDS built with a ring cavity at $1550 \mathrm{~nm}$ are shown in Fig. 2, 3 and 4 along with simulation results, indicating a reasonable fit. The experimental setups are similar to those in $[3,4]$. It is to be noted here that, the absorption coefficient of cane sugar dissolved in distilled water to make the aqueous solution is assumed to be $1 \mathrm{~cm}^{-1}$ for $1 \%$ solution to obtain this fit. The inset in Fig. 3 and 4 indicate that the slope of $\tau-n_{2}$ curve changes with $n_{2}$. For a typical experiment with measurement uncertainty of $4.6 \mathrm{~ns}$ and $450 \mathrm{~ns}$ for conventional and amplified CRDS respectively, the sensitivity changes from $5.93 \times 10^{-3}$ RIU to $1.93 \times 10^{-3}$ RIU for conventional CRDS and $1.10 \times 10^{-2}$ RIU to $1.19 \times 10^{-3}$ RIU for amplified CRDS. This confirms that the sensitivity depends on the region of operation.

\section{Conclusion}

A simulation model is presented to study the effect of change in surrounding refractive index for various taper waist dimensions for a CRDS configuration. It is found that the smaller diameters and longer lengths provide better sensitivity. The loss induced by the sample varies as a quadratic function whereas ring-down time varies as rational function of the refractive index. As a consequence, the measurement sensitivity is found to change with the surrounding refractive index.

Acknowledgement: The authors acknowledge the funding from the People Programme (Marie Curie Actions) of the European Union's Seventh Framework Programme FP7/2007-2013/ under REA grant agreement no. 318941.

[1] Z. Tian, S. S-H. Yam and H. P. Loock, "Refractive index sensor based on an abrupt taper Michelson interferometer in a single-mode fiber," Opt. Lett. 33, 1105 (2008).

[2] P. Wang, G. Brambilla, M. Ding, Y. Semenova, Q. Wu and G. Farrell, "High-sensitivity, evanescent field refractometric sensor based on a tapered, multimode fiber interference," Opt. Lett. 36, 2233 (2011).

[3] D. Wu, Y. Zhao and Q. Wang, "SMF Taper Evanescent Field-Based RI Sensor Combined With Fiber Loop Ring Down Technology," IEEE Photonic. Tech. L. 27, 17, 1802 (2015).

[4] X. Liu, Q. Wang, C. Li, C. Zhao, Y. Zhao, H. Hu and J. Li, "Refractive index sensor based on fiber loop ring-down spectroscopy," Instrum. Sci. Technol., just-accepted (2015).

[5] Z. Wei, Z. Song, R. Song, X. Zhang and Z. Meng, "Measurement of the optical absorption coefficient for liquid based on optical microfiber," Optik 125, 12, 2880 (2014).

[6] A. W. Snyder and J. D. Love, Optical waveguide theory (Chapman \& Hall, London, 1983), Chap. 12.

[7] T. A.Birks and Y. W. Li, "The shape of fiber tapers," J. Lightwave Technol. 10, 4, 432 (1992). 